\title{
LA CONSTITUCIÓN DE LA SOCIOLOGÍA COMO CIENCIA*
}

\author{
Octavio Uña Juárez**
}

\section{RESUMEN}

En este breve estudio se presentan, de manera esencial y sucinta, las aportaciones teóricas clásicas a la constitución de la sociología como saber fundado: Durkheim, M. Weber, Pareto, Simmel, Scheler, L. von Wiese. Líneas argumentativas que, junto con el pensamiento de los "fundadores", marcaron la constitución de la sociología como ciencia. Se pretende mostrar tan sólo el hilo conductor de la "sociología académica".

\section{PALABRAS CLAVE}

Durkheim. Weber. Pareto. Simmel. Scheler. Wiese. Sociología como ciencia. Sociología académica.

\section{ABSTRACT}

In the current research we present, in a basic and concise way, the classical theoretical contributions to the building of sociology as founded knowledge: Durkheim, M. Weber, Pareto, Simmel, Scheler, L. von Wiese. These are different ways of thinking that, together with the "founders" way of thinking, were a landmark for the building of sociology as a science. We intend to show the thread of "academic sociology".

\section{KEY-WORDS}

Durkhein. Weber. Pareto. Simmel. Scheler. Wiese. Sociology as a science. Academic sociology.

*Conferencia pronunciada en el Instituto de Investigaciones Sociales (UNAM) el día 14 de septiembre de 2005, en el LXXV Aniversario de su fundación.

** Catedrático de Sociología de la Universidad Rey Juan Carlos y presidente y miembro fundador de la ACMS. 


\section{INTRODUCCIÓN}

Pretendemos tan sólo mostrar el hilo conductor de la sociología como saber fundado, su empeño teórico por constituirse en conocimiento científico. No es nuestro objetivo -como no lo ha sido al reflexionar sobre los orígenes y la formación en otros estudios nuestros- una presentación exhaustiva de las aportaciones a la teoría y a la metodología en la progresión histórica de los saberes. Tampoco la lectura de estos "clásicos" desde predeterminaciones varias, como la reiterada y nada feliz del conflicto y el consenso. Es ésta una somera referencia a la así llamada sociología académica, de Durkheim a Simmel, la cual, junto con los "fundadores", marcará ineludiblemente la construcción categorial de la sociología. La larga sombra de Kant, el formalismo, la historia y la economía van a precipitar en la elaboración de un grave y original cuerpo temático y metodológico. Sobre este tiempo - digamos, con Aron, "etapa"- pesa como lema íntimo aquel diagnóstico de Fichte sobre la "edad contemporánea": "No reconocer como válido absolutamente nada más que lo que ella concibe" . Un brillante analista de la historia y de la historia de las ideas nos dibuja así la producción en torno a la sociología, que arranca de la segunda mitad del siglo XIX: "En el proceso que sigue la sociología en la segunda mitad del siglo XIX se pierden ideas decisivas contenidas ya en los primeros sistemas de la nueva ciencia. Ello es, podría decirse, el precio que tiene que pagarse para elevar la sociología a una ciencia sistemática según el modelo de las ciencias anteriores, y para ampliar su horizonte a la totalidad de los hechos sociales. Lo que se pierde es la estricta limitación a los problemas del presente, característica en la doctrina de los primeros sociólogos, así como, consiguientemente, el seguro sentimiento de la situación histórica y aquella conciencia de su misión que habría distinguido a la sociología en la hora de su nacimiento"2. Desde Saint-Simon, Comte, Spencer, Marx, Durkheim, Pareto y Weber hasta el presente va el hegeliano -que es virgiliano- "tantae molis erat seipsam cognoscere mentem" de la sociología: el denuedo por su afianzamiento como saber específico, como conocimiento válido y fiable. La sociología sobre y ante sí misma ${ }^{3}$.

\section{DURKHEIM}

Durkheim establece, en su famoso artículo "Representaciones colectivas" (1989), que "la conciencia colectiva es algo diferente de un simple epifenómeno de su base morfológica". E igualmente redunda en su tesis mayor: "cuando se ha constituido un primer fondo de representaciones, éstas se convierten en realidades parcialmente autónomas que viven una vida propia". La vida social queda definida por una "hiperespiritualidad" y "la psicología colectiva es la sociología íntegra"4. En estas afirmaciones y en otras muy similares Durkheim parece alejarse de la relación entre el sustrato y las representaciones colectivas, temario central de la 
sociología del conocimiento, hasta persuadirnos de que éstas "tienen como causas próximas otras representaciones colectivas, no tal o cual carácter de la estructura social". No obstante, Durkheim no llegó a considerar a dichas representaciones como "un círculo cerrado", como "flotando en el vacío" y por entero alejadas de la estructura material de la vida social ${ }^{5}$.

En La división del trabajo social ve en las variaciones de la estructura del medio la explicación de las variaciones del psiquismo humano. Los estados de conciencia no serían otros dado un orden diferente de agrupaciones sociales. Por otra parte, Durkheim parece invocar la naturaleza de los grupos como principio explicativo de los estados de conciencia y de las representaciones. No sin razón se ha querido ver una gran cercanía entre las representaciones colectivas y la ideología, entendida ésta en términos marxianos. Aunque Durkheim piensa que la vida social debe explicarse "por causas profundas que escapan a la conciencia", rechaza el marxismo calificándolo de economicismo y epifenomenismo sociológico. Durkheim acentúa en varios escritos el hiperespiritualismo de la vida social al hablar de "la facultad de ideal", pensando que se minusvalora la vida social cuando ésta es reducida a un cuerpo organizado, sin notar que en ese cuerpo vive un alma, conjunto de los ideales colectivos: "la sociología se coloca de golpe en el ideal. No llega a ese punto lentamente, al término de sus investigaciones: parte de ese lugar. El ideal constituye su propio dominio" .

Durkheim abunda en la relación entre representación y sustrato. El lugar más caracterizado para él es el de la religión, ya que ésta es la madre de la filosofía y de las ciencias, origen de las categorías del pensamiento y tejido de la vida social, en donde acontecen los "primeros sistemas de representaciones que el hombre se ha hecho del mundo". Y si bien los contenidos de la conciencia social están relacionados con los elementos sociales, los universos simbólico-religiosos, el mito, la leyenda, los sistemas cosmogónicos parecen tener una vida propia y autónoma, de modo que no se vinculan de manera directa con determinadas particularidades de la morfología social.

La "sociedad" es la fuente de todos los valores, ella es el origen de la verdad. Las representaciones colectivas, por el mero hecho de serlo, revisten garantías de objetividad. Durkheim y su escuela se moverán en una ambivalencia constante explicando la formalidad del conocimiento por las condiciones sociales y relacionando en otras ocasiones el contenido mismo del conocimiento con la estructura social. Al acentuar el primer aspecto, quiere deducir la universalidad y la necesidad de la razón, la constancia de las categorías y la permanencia de los conceptos por su origen social. Las ideas se organizan sobre y desde un modelo construido y proporcionado por la sociedad. Las relaciones sociales han sido el prototipo de las relaciones lógicas. Esta pretensión de impersonalidad y formalidad del conocimiento es contravenida por el mismo Durkheim al hablar de la transformación del derecho, de los conceptos de lo sagrado y de lo divino y de las ideologías sociales, al 
presentarlos como un simple calco de determinados elementos sociales. Será la sociedad, como lo fuera para sus precursores - Montesquieu, Saint-Simon, Comtela fuente de todos los valores. Queda en la imprecisión, pues, la relación entre conocimiento, representaciones colectivas y condicionamiento social. Igualmente, permanece sin clarificación la específica diferencia de esa conexión por lo que respecta a la religión y a la ciencia ${ }^{7}$.

El propósito de E. Durkheim a lo largo de toda su producción intelectual es la búsqueda de una identidad y especifidad propias para la sociología. Destaca por su contribución al esclarecimiento del objeto y metodología del quehacer sociológico frente a otras disciplinas, sobre todo la psicología. Durkheim significó el salto del organicismo positivista -Comte, Saint-Simon, Spencer- a una teoría auténticamente sociológica: la estructural-funcional. Un salto que supuso la superación de las explicaciones pseudocientíficas, ofrecidas por los planteamientos evolucionistas, que en última instancia recurrían a postulados metafísicos, y de las descripciones históricas que buscaban leyes históricas que mostraran la génesis de los fenómenos, cifra de la explicación racional. Frente a estos planteamientos, Durkheim buscará la explicación sociológica en la estructura interna, como buen heredero de los planteamientos del fisiólogo Claude Bernard, mas superando los razonamientos analógicos.

Para Durkheim el objeto de la sociología es el hecho social. Y por tal entendía: "unas maneras de pensar, de obrar y de sentir, externas al individuo y dotadas de un poder coercitivo en cuya virtud se le imponen" y que además es "general en el conjunto de una sociedad, conservando una existencia propia, independiente de sus manifestaciones individuales" ${ }^{\prime}$. El objeto de la sociología son los hechos sociales, que poseen dos características, exterioridad y constricción. El hombre nace en una sociedad dada a la que ha de adaptarse. Las realidades sociales son "cosas" formadas, el individuo las encuentra así y no puede cambiarlas; los hechos sociales existen independientemente de las conciencias individuales. El lenguaje, la religión o las normas jurídicas no son creaciones de personas particulares; una vez formados, configuran los límites del mundo. Así, lo social y lo individual se entrecruzan en las acciones y los pensamientos, pero lo social viene de fuera y se impone sobre la voluntad particular. Es más, los hechos sociales manifiestan su carácter constrictivo cuando queremos resistirnos a ellos.

Durkheim distingue entre hechos sociales morfológicos (sustrato, estructura, manera de ser) y hechos sociales funcionales (representaciones colectivas, emociones, tendencias colectivas, maneras de hacer), que no implican naturalezas distintas, pues ambas participan de la coercitividad y de la exterioridad. Además, la "solidaridad" entre "órgano y función", entre "causa y efecto" advierte de una mutua dependencia recogida en el concepto de estructura funcional. "Una estructura funcional, será, pues, un sistema de recurrencias sociales o un sistema de fenómenos relativamente estables, cuya condición causal, en cuanto ha de buscarse en la 
constitución del medio social, es la perduración de tal medio en cuanto organización estable de la interacción social, en cuanto estabilidad estructural"10. Un hecho social será funcional "en tanto satisface alguna de las necesidades definidas por el proceso de adaptación social-funcionamiento, que al par es un proceso de estructuración, de consolidación de una cierta estructura. Normas de ser y de hacer se exigen recíprocamente" ${ }^{11}$. Así la funcionalidad significa participar positivamente en el establecimiento, desarrollo y estabilidad de una determinada estructura social.

Los paralelismos entre organismo social y biológico se acaban al tratar el carácter diferencial de la duración, es decir del tiempo, vinculado muy estrechamente al equilibrio funcional. A diferencia del organismo biológico, el sistema de las diferentes funciones, unidas por relaciones definidas, no implica en el organismo social un automático equilibrio funcional. "La interdependencia orgánica no lleva consigo sin más su concurrencia armónica; la "anomía", como falta de regulación interfuncional ("solidaridad orgánica"), el conflicto social, como consecuencia violenta, son hechos posibles dentro del sistema social siquiera se definan como hechos "anormales", en cuanto impiden o perturban el pleno desarrollo de tal sistema según su "tipo morfológico"'2 . La estructura social no determina la duración del sistema social que depende del proceso histórico de constitución y desarrollo de dicha estructura social y de "la duración singular de ciertas recurrencias sociales [que] va estructurando esa totalidad de interacciones". Así, el tiempo social "es entendido como el ámbito en el que se definen los distintos sistemas de equilibrio que son los tipos sociales normales. El acontecer social es, ante todo, proceso de adaptación, de integración social. La solidaridad, y no el conflicto, es el ángulo en el que las relaciones sociales cobran relevancia estructural-funcional"13 .

Una vez definido el objeto de la sociología, los hechos sociales, exteriores y coactivos, es necesario establecer un método propio. Dos son las reglas básicas: "tratar a los hechos sociales como si fuesen cosas", lo que significa que son exteriores al sociólogo, que debe construirlos y arrancarlos del sentido común. La segunda regla es la de que los hechos sociales deben explicarse por otros hechos sociales. Lo social ha de explicarse por lo social, lejos de las reducciones psicológicas y de las analogías biologistas ${ }^{14}$. El carácter objetivo de los hechos sociales les hace ser independientes de las inclinaciones subjetivas y de las prenociones del científico. En el estudio de la sociedad, se ha de adoptar una postura emocionalmente neutra. Sobre ello abundamos más adelante.

\section{MAX WEBER}

El historicismo diltheyano y el neokantismo de Marburg van a indagar en los supuestos teóricos de la ciencia empírica, rechazando así el empirismo, el mecanicismo y el materialismo en los que viene a concretarse el viejo positivismo 
de Comte. La "crítica de la razón histórica" reclama autonomía para las "ciencias del espíritu" y un objeto propio de análisis -el ámbito de la historia, el hombre y sus creaciones-, invocando la prevalencia de lo singular a la vez que se otorga prioridad a la psicología frente a la sociología. "Por esto son falsas las teorías sociológicas y la filosofía de la Historia, que ven en la descripción de lo singular mera materia prima para sus abstracciones"15.

Dicho objeto está presidido por intenciones y vinculado a valores. Además, la comprensión conlleva una "tendencia práctica en juicios, ideales y normas". En las ciencias del espíritu "el estudio de los fundamentos explicativos de los juicios acerca de la realidad está ligado al de los fundamentos explicativos de los valores e imperativos, que están destinados a regular la vida del individuo en la sociedad"16. Así se articulan ciencias normativas y ciencias empíricas, siendo lo propio de las ciencias sociales la aprehensión de la organización externa de la sociedad así como lo es de la hermenéutica el descubrimiento de significados para la comprensión de la singularidad de la naturaleza humana en la historia.

Para el neokantismo, especialmente de la mano de Windelband y Rickert, el conocimiento que trata de sorprender la singularidad y unicidad de los objetos de carácter idiográfico, fundamento de las ciencias de la cultura, es tan válido como el conocimiento generalizador, de carácter nomotético y fundamento de las ciencias de la naturaleza ${ }^{17}$. De la estructura misma de la conciencia, de su trascendentalidad $\mathrm{y}$, por tanto, de su imposibilidad de abarcar la totalidad de lo real, nacen estas ciencias que acometen el conocimiento de ámbitos diferenciados de la realidad. La sociología centrará su objeto en la identificación y selección de valores con el propósito de aislar y perfilar la individualidad significativa, dado que la cultura se asienta en el valor: “... la sociología, como tal disciplina autónoma, resultaba imposible. Las categorías centrales de acción y relación social se desvanecían en esa universal definición del mundo a partir de la pura conciencia subjetiva, que se puede definir concretamente como totalidad de los hechos de la conciencia (Dilthey)... o, universalmente, como sujeto trascendental constituyendo normativamente sus objetos (Rickert)"18.

En este contexto -y con una actitud en parte de aceptación y en parte de rechazo al positivismo, al historicismo y al marxismo- elabora M. Weber su obra.

Captar el pensamiento de M. Weber no es asunto fácil, como lo prueban las múltiples y contradictorias interpretaciones a que ha dado lugar en la abundante bibliografía posterior que ha suscitado ${ }^{19}$. Este hecho está motivado por las siguientes razones. 1) Como el mismo Weber sostiene: "Nuestro propósito aquí no es el de ofrecer soluciones, sino el de expresar problemas" ${ }^{20}$. Weber, como Heidegger o Wittgenstein, no concretó en una sistematicidad definitiva sus ideas. 3) El pensamiento de Weber no es "monolineal", dado que arranca de una situación cultural compleja.

Para detectar las fuentes principales del pensamiento weberiano, es preciso tener en cuenta que Weber es a la vez un sociólogo y un filósofo de la cultura. Y 
que en tanto pretendía ser lo primero en cuanto era determinado por lo segundo. Entre sus fuentes sociológicas cabe mencionar representantes insignes de la sociología positiva "clásica" -Comte, Durkheim, J.S. Mill, Pareto, March y Poincaré, en suma, una sociología que se centra en la constatación de hechos empíricamente observables, susceptibles de "formulación" legal, según nexos causales análogos a los de la ciencia empírica. El intento de Weber es no tanto rechazar esta concepción de la sociología, cuando reformarla y completarla. Lo social es para él un nuevo mundo, hasta cierto punto autónomo por relación a lo natural. Por ello desplazó la sociología de las ciencias naturales al ámbito de las que llamó "histórico-culturales o sociales". En sociología es preciso aunar constatación y comprensión, ya que la objetividad, más allá de los simples hechos, se refiere a un obrar con sentido subjetivo, susceptible, más que de simple formulación legal según el modelo causal, de interpretación comprensiva de significado y sentido. A esta nueva manera de entender la objetividad, deberá corresponder una nueva teoría heurística según nuevos modelos conceptuales. Las consecuencias de esta "revolución en sociología" llegan hasta nuestros días por la preocupación de la autonomía del juicio sociológico y del razonamiento metodológico.

Las fuentes filosóficas de M. Weber, al plantearse la cuestión de la estructura y función de la ciencia social, arrancan del debate entre historicismo y neokantismo en torno al concepto de "ciencia cultural". Así, del neokantismo de orientación cientificista tomó Weber el alcance histórico-crítico del razonamiento de carácter científico en sociología. De Dilthey, tres aspectos influyen en él: a) la distinción entre ciencias de la naturaleza y ciencias del espíritu, que Weber transformó en ciencias histórico-culturales e histórico-sociales; b) el concepto de "Verstehen", que Weber retomó y aceptó, aunque rechazó el intuicionismo de Dilthey, aduciendo que el empleo de un método de comprensión no excluye la explicación causal o formulación de hipótesis interpretativas verificables causalmente de forma empírica; y c) el descubrimiento deltheyano de la dimensión histórica y su correspondiente componente cultural en el ámbito humano. De H. Rickert, Weber tomó los conceptos de "ciencia cultural" y de "relación de valor". Para Weber, las condiciones de la objetividad de la ciencia social son: a) por una parte, de carácter negativo, la neutralidad valorativa se articula sobre la base de la distinción entre juicio de valor y relación de valor; b) por otra parte, de tipo positivo, la verificación causal como garantía de la validez del conocimiento (con lo que se opone a la validez incondicional de los valores de Rickert) ${ }^{21}$.

En Weber se observa una transición desde los primeros escritos metodológicos a la posición formulada en Economía y sociedad. Primeramente, plantea la subordinación de las ciencias de la cultura a la historia y su nucleación en torno al método ideográfico. Finalmente, defiende la autonomía de la sociología como ciencia que estudia las uniformidades de la conducta social dotadas de sentido, es decir, la sociología como ciencia generalizadora. 
Para Weber, la sociología es una ciencia de la realidad en perspectiva cultural. A diferencia de las ciencias naturales, a la sociología le interesan los aspectos individuales bajo su sentido cualitativo. Como ciencia cultural, se mueve en el terreno de lo significativo que, en cuanto tal, no coincide con ninguna ley. Y ello se debe a que "el concepto de cultura es un concepto de valor"22. Premisa implícita en toda ciencia cultural es que el hombre es ser civilizado que, mediante actitudes conscientes de su voluntad, se halla capacitado para conferir un sentido al mundo. Por consiguiente, el conocimiento de la realidad cultural "es siempre un conocimiento bajo nuestro punto de vista específicamente particular"23.

Pero la realidad del mundo, en opinión de Weber, es irracional en sí misma y su devenir es un acontecer caótico. Sólo la racionalización es capaz de conferirle un sentido. Además, la realidad posee infinitos aspectos a los que el conocer y los conceptos sólo pueden llegar de modo aproximativo y por selección de aspectos sucesivos. A ello se une el problema de captar la realidad social, significante sólo en la interrelación individual.

Para Weber, el objeto de la sociología es la acción social. Este concepto significa, por una parte, acción como conducta significativa para el sujeto (con un sentido subjetivo de relacionar la realidad con el mundo de los valores) y, por otra, acción social con dimensión de alteridad (con un sentido referido a la conducta de los otros, orientándose por las expectativas ajenas). Así, este concepto implica la no reductibilidad de la conducta humana a la biología (darwinismo social), psicología (Freud), sociología (positivismo y marxismo). El sujeto sólo puede ser la persona individual. Y presupone la existencia de un sistema de significaciones y valores comunes (la socialización). Pese a los tipos de acción social existentes, lo común es que se den uniformidades de conducta social. Estas uniformidades se diferencian por el nivel de claridad y determinación del sujeto. La tipología de la acción social aparece, así, como la ordenación de la conducta en una escala graduada de significación.

Sostiene igualmente que la finalidad de la sociología es comprender la acción social para explicarla causalmente. En este caso, el conocimiento por la generalización no alcanza la particular individualidad de lo social y su acontecer histórico. Por otra, el método individualizador carecería del vigor que exige el saber científico al olvidar lo genérico. Por consiguiente, el método de la sociología debe presentar dos aspectos determinados por la bipolaridad del objeto: la comprensión y la explicación, para hallar, respectivamente, el sentido y la regularidad. Sólo la comprensión unida a la explicación deviene en interpretación causal válida: "Dar una interpretación causal correcta de una acción particular es concebir como apropiado el curso externo de esa acción y su motivo al tiempo que ambos se entienden como relacionados entre sí de una manera cuyo significado sea comprensible"24.

Lo distintivo de la comprensión es que toda interpretación tiende a la evidencia. La evidencia sociológica deriva sólo de la relación con los valores. En esto Weber 
se separa de Durkheim, al considerar que el objeto de la investigación sociológica es el sentido de las actividades reales y concretas, a donde no llega el simple método de las conexiones causales externas. La evidencia de la comprensión puede ser endopática o racional. La segunda es la más universal. Por ello la sociología utiliza la acción racional con arreglo a fines como modelo para construir leyes y conceptos (la racionalidad funcional es la racionalidad constituyente de la lógica constructiva). Pero la evidencia de una interpretación no garantiza su validez causal, es sólo una "hipótesis causal particularmente evidente". Puede no haber coincidencia entre la interpretación elaborada y la conexión de sentido efectiva, pese a la evidencia de la primera, debido a diversas causas. ${ }^{25}$ Así pues, la sociología de Weber se puede definir como "la ciencia cuyo objeto es comprender por interpretación la actividad social para explicar luego causalmente el desarrollo y los efectos de esta actividad" 26 .

El corpus teórico de la sociología, para nuestro autor, está formado por un conjunto sistemático de leyes generales (de valor probable) e ideas-tipo, que intentan explicar las uniformidades de conducta social dotadas de sentido.

a) Las leyes sociológicas son probabilidades comprobadas de modo que, dadas ciertas condiciones efectivas, ciertas acciones sociales -típicamente comprensibles por sus motivos-, sucedan en la forma esperada. Su elaboración tiene tres momentos. 1) Observaciones de la experiencia y determinación de los hechos importantes para la investigación. Este proceso viene determinado por el punto de vista del observador, que, mediante la "relación de valor", selecciona entre la multiplicidad empírica. 2) Elaboración de la hipótesis interpretativa. Construcción de un curso de conducta, coherente lógicamente en su estructura interna, de acuerdo con los hábitos sociales medios o típicos, lo que lo hace particularmente evidente. Este momento, que Weber llama "adecuación de sentido", implica ya la adecuación causal. 3) Control empírico de las hipótesis. Verificación de la probabilidad de que siempre ocurra de la misma forma ${ }^{27}$. Esta adecuación causal, es, a la vez, una adecuación de sentido. El "valor explicativo" es sólo probable, porque, pese a lo señalado referente al objeto de la comprensión (optar el sentido subjetivo mentado por el sujeto), lo que se capta es el sentido objetivo (empíricamente válido). El sujeto no es real, sino típico y la racionalidad de las leyes sociológicas es una racionalidad con arreglo a regularidades.

b) Las ideas tipo. Son construcciones elaboradas mediante la acentuación unilateral de determinados rasgos de la realidad observada y que forman un cuadro abstracto lógicamente coherente, sirviendo como instrumentos para ordenar conceptualmente la realidad. El ideal-tipo, pues, no es realidad empírica, sino que se alza sobre ella para dominarla mejor teóricamente. Es instrumento y no objeto ni fin del conocimiento. En la búsqueda de la objetividad del conocimiento sociológico, el único fin perseguido por un tipo ideal, cualquiera que sea su contenido, es "comparar con él la realidad empírica, para determinar su contraste, su diferencia 
o su relativo acercamiento, con el fin de poderla describir con unos conceptos lo más unívocamente comprensibles y comprenderla y explicarla gracias a la atribución causal"28. Respecto a su naturaleza, un tipo ideal recoge los rasgos específicos y acentuados de una realidad individual comparada con un modelo conceptual ideal, pero es algo muy distinto de un juicio de valor. Se trata de una construcción mental, para la caracterización sistemática de las relaciones individuales, significativas por su singularidad o "relación significante"29.

Tanto los conceptos-tipo como las leyes son tipos ideales que revisten un valor heurístico, teniendo como finalidad explicar el desarrollo real de los hechos a partir de la relación de éstos con aquellos: "poseen un alto valor heurístico para la investigación y un enorme valor sistemático para la representación si sólo se las utiliza como medios conceptuales para medir la realidad y compararla con ellas" ${ }^{\prime 30}$. Este carácter típico-ideal es afirmable no sólo externa sino internamente.

Un punto discutido y diversamente interpretado en Weber es la neutralidad axiológica. "Weber no defiende la asepsia valorativa de la actividad científica, sino que ataca la confusión entre lo cognitivo y lo valorativo. Rechaza tanto el que se inscriba la ciencia en una tendencia partidista como el que se la "descaracterice". No sólo no se opone a que el científico profese determinados ideales, sino que afirma que está obligado a defenderlos. Pero exige que se hagan explícitos, y que se marque expresamente el tránsito de un plano del discurso (el de los hechos) a otro (el de las valoraciones personales)" ${ }^{31}$. Weber se expresa así: "Creemos efectivamente que una ciencia experimental nunca podrá tener por tarea el establecimiento de normas e ideales, con el fin de derivar de ellas unas recetas para la "praxis". ¿Qué se deduce de esta afirmación? En modo alguno que los juicios de valor se sustraen a toda discusión científica... pero la crítica no se detiene ante los juicios de valor. Por lo tanto, la pregunta debe formularse más bien de la forma siguiente: ¿Qué significa y qué se propone la crítica científica de ideales y juicios de valor?"32.

El tema de los juicios de valor en las ciencias humanas era vivo en tiempo de Weber, renacido por una fuerte polémica que le alcanzó de lleno, obligándole a tomar posición. Para esclarecerla, distingue J. Freund un doble plano en la actitud de Weber, el pedagógico y el de la investigación. Con respecto al primero, Weber propone una equilibrada ética profesional a todo aquel que dedicado a las actividades académicas intente cumplir su cometido científico honestamente. Exige a todo maestro conciencia clara de la heterogeneidad entre ciencia y convicción, guardándose sus valoraciones personales sobre lo que enseña, pero también resistiendo a toda imposición por parte de los políticos o de los grupos de presión. En último extremo, tal valoración debe ser oportuna y arrancar de una distinción neta entre razonamiento lógico y valoración práctica.

Pero es el plano de la investigación el que ocupa primordialmente a nuestro autor. Es necesario distinguir en él entre referencia a los valores y juicios valorativos. 
Considera a la sociología como una ciencia empírica de estricto rigor gnoseológico y crítico. Pero no conseguirá este objetivo por una mera consideración de hechos sociales y su formulación legal. Sin negar este aspecto causal, Weber cree que la investigación sociológica recobra su especificidad y autonomía metódica mediante la comprensión individual de acciones significativas dentro de una constelación valórica, marcada por las intenciones y los fines. Por eso cabe concebir la sociología como análisis de actividades o análisis científico de los medios en orden a un fin dado, preguntándose por la idoneidad de tales medios. Una decisión personal puede ser iluminada por la ciencia cuando ésta oriente su elección de fines. Cabe, pues, un estudio científico de los juicios de valor en este sentido, que ayuda, además, a enjuiciar críticamente. Se trata, dice Weber, de una critica en forma de juicio lógicoformal sobre el contenido formal de los juicios de valor y las ideas históricamente dadas. La utilidad de tal critica reside en que ayuda a reflexionar sobre axiomas últimos en que se basa el contenido del querer y, además, sobre las escalas de valor último e inconsciente, pero necesarias.

De este modo, la sociología, como análisis de lo real, debe incluir los valores a que van referidas las acciones humanas. Los valores son, en este sentido, dimensión inseparable de la realidad sociológica. Sin embargo, relacionar hechos con valores según un cuadro mental, ideal-tipo, es distinto de efectuar la "apreciación evaluada de esa realidad a partir de ideales" ${ }^{\prime 33}$. Para no caer en lo segundo en nombre de lo primero, exige Weber el "deber elemental del control científico". El confundir, en diversas ciencias, lo real con lo normativo, ha llevado a una degradación del trabajo técnico y científico. Con ello Weber intenta devolver a la sociología su aspecto crítico e instalarla dentro de sus propios límites noéticos y reales, a los que habrá de ajustarse su instrumento metódico.

La consideración weberiana de la sociología como ciencia empírica implica que ésta se mueve en el ámbito de la constatación racionalizadora de la actividad relacional humana significante. Intentar fijar un cuadro valoral concreto o emitir un juicio de preferencia entre varios equivaldría a transgredir sus propios límites. Y la razón es clara: "Una ciencia experimental nunca podrá tomar por tarea el establecimiento de normas o ideales" ${ }^{\prime 34}$. Las ciencias sociales deben proponer demostraciones científicas, universalmente admisibles, pero no están capacitadas para "deducir de forma unívoca unos contenidos culturales de carácter obligatorio".

"Sólo las religiones positivas o sectas dogmáticas son capaces de conferir al contenido de los valores culturales la dignidad de imperativos éticos de una validez incondicional" ${ }^{35}$. De ahí que Weber crea urgente una abstención de todo juicio valorativo en las ciencias empíricas: "La constante mezcla de investigación científica de hechos y de razonamientos valorativos es una de las características más difundidas, pero también más perniciosas, en los trabajos de nuestra especialidad"36.

Weber insiste en ello a propósito de los ideales típicos, afirmando que un tipo ideal es diferente de la apreciación evaluadora. Según su juicio, las discusiones 
sobre los valores no son estériles, pues se hallan al servicio del conocimiento de la verdad. Considera la ciencia empírica incapacitada para investigar el sentido de las valoraciones y para asignar a un valor determinado un lugar dentro de la esfera total de los valores. Esta sería misión de la filosofía.

Libertad de valoración y referencia a los valores son los quicios sobre los que Weber hace girar el método sociológico.

Respecto a las "referencias al valor", Weber remite a sus estudios anteriores y a los trabajos de H. Rickert. La referencia a los valores es un eficiente instrumento lógico de investigación sociológica en tres aspectos: 1) domina en la selección de una determinada temática; 2 ) orienta en la formación del objeto de un estudio empírico y 3) ofrece las problemáticas necesarias para su trabajo. Pero todo esto se distingue del juicio valorativo. Dentro de los estudios empíricos esta situación puramente lógica no legitima ningún tipo de valoraciones prácticas. A pesar de ello, en concordancia con la experiencia histórica, dicha situación pone en evidencia que los intereses culturales o de valor indican la dirección del trabajo puramente científico-empírico. Está claro que las discusiones sobre el valor pueden constituir el medio de expresión de tales intereses axiológicos en su casuística. Incluso pueden facilitar en gran medida el trabajo del investigador científico, en especial del historiador ante todo el campo de la "interpretación de los valores", que constituye una de las principales tareas del trabajo empírico propiamente dicho.

En todo caso, recuerda Weber que se debe distinguir entre "valoración y referencia a los valores" y también entre "valoración e interpretación de los valores". En cuanto a esta última, el "desarrollo de las posibles tomas de posición significativas frente a un determinado fenómeno"37. Las soluciones unívocas en la ciencia empírica sólo son posibles allí donde las estructuras que las condicionan están ya formalmente dadas.

Finalmente, Weber se enfrenta con la previsible objeción: es imposible pensar en una teoría axiológica neutra en este campo especial de la ciencia. Su respuesta, prolija, se resume en esta rotunda afirmación: "Como se comprenderá es completamente falsa. Tan falsa, que precisamente la libertad de valores es el presupuesto de todo estudio científico sobre la política y, ante todo, de la política social y económica" 38 . En otros términos: "el sociólogo tiene por misión analizar las estructuras de la sociedad, la naturaleza de las convenciones, del derecho, de la política y de la economía, no juzgar al reformador social o decretar cuál es la mejor sociedad" 39 . Tras esto, cabe estructurar la respuesta weberiana a la sociología como ciencia libre de valoraciones en los siguientes puntos, a) La sociología puede ocuparse de los valores y estudiarlos científicamente ("crítica técnica"), dado que son objetos culturales. b) Pero no puede deducir la validez de los valores, asimilando el ser con el deber ser. c) Es difícil evitar que los valores impregnen la investigación del científico social. d) La sociología precisa el uso constante de los valores, desde la relativa óptica metodológica e instrumental. En síntesis, Weber concretó el 
valor y la función del conocimiento sociológico así: la sociología es una ciencia empírica, estableciendo lo que de hecho ocurre y no lo que debe ocurrir. No tiene un sentido normativo. Su valor reside en que ilustra sobre las condiciones, posibilidades, consecuencias y verdaderos fundamentos de nuestras acciones. Por lo tanto, se trata del nivel de conocimiento superior de la acción social dentro de una escala que integra, en su extremo inferior, las formas cotidianas de comprensión.

Por otra parte, nuestro autor afirmó que la estructura de la sociología está relacionada con las formas de interacción social. En concreto, el carácter típicoideal del cuerpo teórico de la sociología y su estructuración en torno a los valores de la generalidad de su conceptualización y de la validación empírica están relacionados con las formas típicas de acción social de las modernas sociedades industriales, que se orientan por cuatro máximas fundamentales: la neutralidad afectiva, el universalismo, la relación adquisición-prestación y la especialización. Una acción social de estos caracteres formales es la que corresponde a la racionalidad material del "cálculo de capital" ${ }^{40}$. Estas formas de interacción social se basan en estructuras tipificadas de expectativas y roles. La sociología weberiana sería, así, la representación de la conciencia del hombre de esta sociedad en su más alto nivel de claridad y determinación, en la medida en que fundamenta sus tipificaciones en probabilidades objetivas. De otro modo, en la medida en que opera sobre la base de una racionalidad con arreglo a lo regular.

\section{PARETO. SOCIOLOGÍAS DEL TIEMPO DE M. WEBER}

Los sociólogos Durkheim, Weber y Pareto comparten, según R. Aron, la conciencia de crisis de la sociedad europea de su tiempo. Como sociólogos creían que la ciencia era el modelo único de pensamiento y "que la sociedad no podía conservar su estructura y su coherencia sin una fe común que reuniese a los miembros de la colectividad"41 . No obstante, su reflexión "acerca de las relaciones entre la ciencia y la religión, o entre el pensamiento racional y el sentimiento, en función tanto de la exigencia de pensamiento científico como de la exigencia social de estabilidad o de consenso" "42 tiene diferente expresión en cada uno de estos autores. Así, Pareto comprueba que si bien desde el punto de vista de la ciencia las conductas sociales son irracionales, son útiles desde el punto de vista social. "Si un sociólogo revela a los hombres el reverso del decorado o da vuelta a las cartas, arriesga destruir ilusiones indispensables. Hay una contradicción entre los sentimientos necesarios para el consenso y la ciencia, y dicha contradicción revela la no-verdad de estos sentimientos" ${ }^{\text {"43 }}$.

Partiendo de la distinción, producto de la abstracción, entre acciones lógicas y no-lógicas, Pareto asigna como objeto de estudio de la sociología la acción nológica, muy en sintonía por una parte con el movimiento irracionalista de finales del siglo XIX, en el que militaban intelectuales como Bergson, Sorel, Freud y James. 
Estos dos últimos y Pareto intentaron explicar racionalmente lo irracional. Por otra parte, con los maestros de la sospecha: Marx, Freud, Nietzsche, que sometieron a crítica las explicaciones que los miembros de una sociedad se dan a sí mismos sobre su propia realidad.

Bajo la influencia de la Ilustración y del positivismo, sostiene la necesidad de clarificar y separar las formas lógicas y no-lógicas de la acción social. Una conducta será lógica cuando la relación medios-fines en la realidad objetiva coincida con la relación medios-fines en la conciencia del actor: "denominaremos actos lógicos a las operaciones vinculadas lógicamente con su fin, no sólo con respecto al sujeto que realiza estas operaciones..., denominaremos no-lógicos a los restantes actos, lo que no significa que sean ilógicos" ${ }^{44}$. Los actos humanos, desde esta consideración, quedan tipificados así (Cuadro 1).

Tipos y especies ¿Tienen las acciones un fin lógico?

Objetivamente

Subjetivamente

$I^{\mathrm{a}}$ Clase: acciones lógicas

El fin objetivo es idéntico al fin subjetivo

Sí

Sí

II ${ }^{\mathrm{a}}$ Clase: acciones no-lógicas

El fin objetivo difiere del fin subjetivo

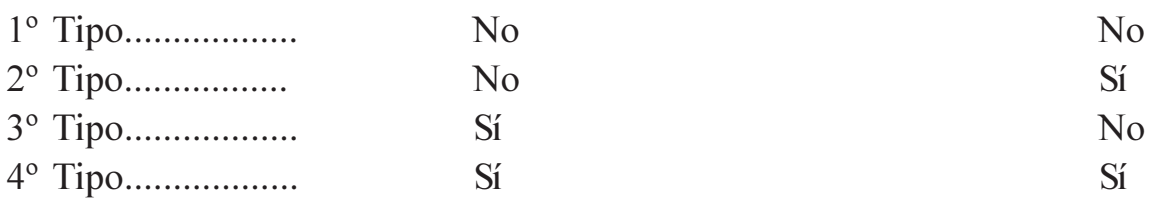

Especies de los tipos $3 .^{\circ}$ y $4 .^{\circ}$

$3 \mathrm{a}, 4 \mathrm{a}$

El sujeto aceptaría el fin objetivo si lo conociera

$3 \mathrm{~b}, 4 \mathrm{~b}$

El sujeto no aceptaría el fin objetivo si lo conociera ${ }^{45}$

Cuadro 1

La acción lógica o racional será el resultado de un razonamiento o estará guiada por intereses, mientras que la no-racional o no-lógica estará gobernada por los residuos, que no hay que confundir con los sentimientos y los instintos, aunque ciertamente corresponden a contenidos que están en la naturaleza de la conducta humana. Los residuos no son realidades concretas sino conceptos analíticos creados 
por el observador para explicar los fenómenos sociales. Hay que distinguirlos, igualmente, de las derivaciones (componente ideológico constituido por doctrinas, teorías, lenguajes, etc.) que son las explicaciones que otorgan logicidad aparente a una conducta que está originada por los residuos, y con ellos justificar las conductas y disimular los sentimientos de los sujetos.

Para el sociólogo, tendrán especial importancia los actos no-lógicos, que, desprovistos de fin objetivo, poseen una finalidad subjetiva; espacio en el que quedarían comprendidas las conductas rituales o simbólicas. Igualmente, aquellos actos en los que no hay coincidencia entre lo subjetivo y lo objetivo, género al que pertenecerían las conductas dirigidas, de algún modo, por errores científicos. Pareto intentará el estudio lógico de la conducta no-lógica y para ello acudirá a un discurso científico, denominado lógico-experimental. Tal proposición epistemológica para la sociología se basa en el análisis de los hechos sociales al margen de su valor intrínseco. Parte de la experiencia de la observación, de los hechos, para elaborar teorías, se afirma como contingente y relativa, juzga de las cosas y no de los sentimientos y procede por aproximación sucesiva. Está presidida por la convicción de que la ciencia tiene un reducido poder sobre la realidad. Si el objeto de la ciencia es el descubrimiento de constantes y uniformidades de la realidad, constatadas experimentalmente, las relaciones entre los fenómenos, la sociología va a quedar entregada al estudio de lo no-lógico.

Como consecuencia de esta suposición teórica y metodológica en torno a los hechos sociales, Pareto va a descubrir los elementos constitutivos de la acción nológica, como son los instintos e inclinaciones humanas. Tales instintos constituyen los "residuos" o constantes psicológicas, encubiertos en las derivaciones, es decir, en el componente ideológico constituido por doctrinas, teorías, lenguajes, etc. "Debemos observar, además, que en el estado actual de las ciencias sociales, que no han alcanzado aún el estado de ciencias lógico-experimentales, la prevalencia de los residuos de la clase es precisamente la prevalencia no sólo de interés, sino también de derivaciones, de religiones intelectuales y no de razonamientos científicos; y esas derivaciones a menudo se alejan de la realidad mucho más que las acciones no-lógicas del simple empírico" ${ }^{46}$. Los residuos, de difícil concreción y delimitación, vendrían a ser los elementos relativamente inmutables de la vida humana. Serían de seis clases: 1) el instinto de las combinaciones, 2) la persistencia de los agregados, 3) las necesidades de la exteriorización de los sentimientos, 4) los relacionados con la vida social, 5) los relativos a la integridad del individuo, 6) el residuo sexual. Por su parte, las derivaciones tendrían cuatro fases, según los distintos tipos de justificaciones que dan los hombres a los sentimientos que expresan: 1) las afirmaciones, 2) la apelación a la autoridad, 3) la armonía con los sentimientos o con los principios, 4) las pruebas verbales.

La sociedad humana, según el teórico italiano, habita en un estado intermedio entre dos "tipos extremos" de sociedades: una sociedad en la que actúan 
exclusivamente los sentimientos, sin razonamiento alguno, y otra en la que, por el contrario, actúan sólo razonamientos lógico-experimentales. Aunque Pareto representa en su reflexión sobre la vida social y política un rechazo a la fe en la razón, un desencanto de la Ilustración y del racionalismo, la crítica al cientifismo y a la pretensión racionalista quiere delimitar el ámbito estricto de la ciencia, señalándole también sus límites. Aunque coincide con Durkheim en la valoración de las creencias y sentimientos en la sociedad, difiere del optimismo racionalista de éste.

Pareto quiere enfatizar en la autonomía de la sociología, a la vez que mostrar que las formulaciones científicas y las explicaciones de la conducta han olvidado los elementos no-lógicos. Esta debe arrancar desde la consideración de los residuos, que son los motivos reales y constantes de la acción social y no de las derivaciones, que son motivos ficticios y variables de la acción. Sólo tales derivaciones son observables y de ellas parte el sociólogo italiano para comprobar realmente las hipótesis establecidas previamente; aunque su metodología es hipotético-deductiva, como en la ciencia natural, evitando las categorías causa-efecto, particularidad y cualidad. Pareto, pues, incluye en la sociología el análisis de los elementos lógicos, de la acción-análisis y la explicación total -síntesis- de la acción concreta, incluyendo el aspecto económico ${ }^{47}$.

Queremos señalar, breve e indicativamente, algunas aportaciones del pensamiento sociológico contemporáneo de Max Weber.

Tönnies, brillante analista de las creaciones de la voluntad humana ("Wesenwille" y "Kürwille") y de las relaciones sociales ("Gemeinschaft" y "Gesellschaft"), propugna una sociología pura o teorética -estática y elaborada con un método constructivo-, aplicada -dinámica y deductiva- y empírica, basada en la inducción ${ }^{48}$. Tönnies quiere construir desde la "sociología formal", aunque también aporta a la sociología psicológica y a la sociología histórica. ${ }^{49}$

Para Sombart, la sociología es la "ciencia experimental sistemática de la convivencia humana" ${ }^{50}$. El conocimiento de la socialidad humana implica "fijación de aquello que era, es o será y, por tanto, exclusión del examen de aquello que debe ser, y así de toda política" 51 . Sombart opone al monismo gnoseológico científico natural, propio de las ciencias de la naturaleza, el pluralismo en los modos de conocer. En concreto, afirma que el modo de conocer específico de las ciencias del espíritu o culturales es la comprensión, entendida como captación del sentido o de la esencia, el saber del porqué. La superioridad de la comprensión respecto al conocimiento natural radica en su inmanencia: "De esta identidad de sujeto y objeto de conocimiento..., nos cercioramos nosotros no por un acto de conocer, sino por nuestro obrar" 52 . Y no hay que equivocarla con la intuición, pues "sólo la determinación por categoría de lo visto es conocimiento"53. Sombart distingue tres tipos de comprensión: 1) Una pura comprensión de sentido "por la cual lo intemporal viene captado en sus manifestaciones históricas" ${ }^{\prime 5}$; 2) una comprensión de cosas 
-para las configuraciones objetivas de la cultura de la sociedad en la historia- y 3) una comprensión anímica o psicológica, para hallar conexiones de acciones.

El intento mayor de Simmel es aunar en una misma perspectiva el intersubjetivismo, ámbito de análisis microsociológico, con el formalismo normativo, ámbito macrosociológico. Ya que toda ciencia está entregada a una alternativa: o "descubrir leyes" o "explicar y comprender procesos singulares históricos y reales" 55 . El centro de este intento se concreta en tipificar las "formas sociales" permanentes: "un análisis del concepto de sociedad que se caracteriza por la distinción entre forma y contenido de la sociedad"56. Por ello, la sociología es una ciencia analítica abstracta y su cometido, la consideración de las pautas de interacción social y las formas abstractas de la vida social, vinculadas ineludiblemente a interpretaciones psicológicas. El objetivo específico de la sociología no será el individuo, sino la sociedad, hecho generado por la interacción social. "Una sociedad existe allí donde varios individuos entran en acción recíproca" ${ }^{57}$. Dicho análisis tiene tres vertientes: general, formal y filosófica. Así lo que en Simmel no era nada más que un recurso metodológico - el análisis de la interacción- se tornará su quehacer mayor y su más sugerente aportación a la teoría, quedando relegado a un segundo plano dicho análisis de la interacción, su propuesta fundamental y originaria $^{58}$.

Para Scheler, la sociología no trata de "hechos ni sucesos individuales", sino de "reglas, tipos (tipos medios y tipos ideales lógicos) y, donde es posible, de leyes" El objeto genérico de su análisis es la vida humana, y el específico el contenido subjetivo de la vida humana misma. Este es estudiado descriptiva y causalmente, pero desde una perspectiva muy peculiar: "su determinación por las formas de unión y de relación sucesiva y simultánea que existen entre los hombres". A lo que llama el filósofo "determinación efectiva" ${ }^{60}$. De este modo, rechaza los "contenidos con sentido weberianos" así como el carácter material de la determinación social de la conciencia. La sociología se divide en pura o apriorística, que acomete esencias, y empírico-inductiva, estática y dinámica, cultural y real. A propósito de esta división, al fundamentar la sociología de la cultura y, al interno de ella, la sociología del saber, precisa Scheler su peculiar entendimiento de la sociología. Así pues, es la intención ontológica predominante de la acción humana, dirigida por ideales o por impulsos, el criterio diferenciador de la sociología cultural o de la superestructura y de la sociología real o de la infraestructura. La primera investiga el ser y el obrar del hombre, que depende de condiciones preponderantemente espirituales: ideales y valores. La segunda atiende a los cambios sociales reales, motivados por los impulsos en la acción humana. Los dos ámbitos son independientes. Según Scheler, las ideas sólo se actualizan en la cultura si están unidas a los intereses e impulsos colectivos e incorporados a las instituciones. Aunque esta división es también metodológica, no deja de ser provisional ni pierde de vista la meta única "de toda sociología actual en el conocimiento de una suprema 
ley de orden de sucesión en la actuación de los factores ideales y reales determinantes del contenido de la vida total de los grupos humanos y condicionados a su vez "sociológicamente", esto es, por las relaciones entre los hombres, las formas de relación y las agrupaciones"

El intento de Scheler es fundar la sociología de la cultura y del saber y, al acometerlo, concreta su peculiar idea de la sociología, que a su vez no se entiende sino desde la radicalidad de la sociología del conocimiento aplicada a todo saber y a toda forma de conocimiento. La sociología del saber investiga la "naturaleza social de todo saber" ${ }^{\prime \prime 2}$. Trátase, pues, de descubrir y describir la conservación y transmisión de los saberes, su ampliación y promoción metódica, el origen-teoría del conocimiento- y la validez del saber -lógica-. A lo largo de varios escritos va a dar cuenta de esta pretensión (Escritos relativos a la sociología y la teoría de la concepción del mundo, 1923; Sociología del saber, 1924; El saber y la cultura, 1925; Conocimiento y trabajo, 1926) Scheler enjuicia así la sociología del saber: "La sociología del saber... tiene por misión indagar las leyes y los ritmos con arreglo a los cuales fluye el saber desde las cumbres de la sociedad (las "elites" del saber) hacia abajo, y cómo aquí se distribuye temporalmente entre los grupos y las capas, mas, asimismo, cómo la sociedad regula organizatoriamente esta distribución del saber..." ${ }^{63}$. También el veredicto de Mannheim sobre tal pretensión y empeño: "El resultado fue un grandioso bosquejo sistemático, lleno de intuiciones profundas, pero carente de un claro método de investigación, adecuado a una ciencia cultural sociológicamente orientada" ${ }^{64}$.

Desde la "sociología formal" desarrolla L. von Wiese su "doctrina relacional", un intento de descripción sistemática de las relaciones y formas sociales. Wiese enfatiza sobre el carácter científico de la sociología ${ }^{65}$. Para ello habrá que liberar a ésta de especulaciones filosóficas y de juicios de valor, también de sus pretensiones novadoras, renovadoras y revolucionarias. Su objeto ha de ser el discernimiento de los procesos relaciónales de socialización, desde el estudio empírico de los hechos sociales. Se trata de aclarar la esencia de lo societario -"das Wesen des Gesellschaftlichen"-, que no pertenece ni a las ciencias de la naturaleza ni a las del espíritu, sino que es un apartado de la doctrina general del hombre- "gesamtlehre von Menschen"-, que estudia el espacio en el que los hombres se unen o separan mutuamente, lo interhumano-"das Zwischen menschliche" aislacionísticamente, no históricamente. Que esta es la "desviación" de la sociología formal, como quiere Freyer: "... la sociedad con la que tiene que ver la sociología, no es una multiplicidad de formas relacionales y fenoménicas que se repiten idénticamente y que, por tanto, pueden ser arrancadas de su lugar histórico sistematizándolas puramente como formas. La sociedad es, más bien, una sucesión irreversible de situaciones totales, a través de la cual corre el curso histórico. Todo fenómeno social pertenece al seno de una situación histórica total de esta especie, y tiene en ella su lugar. La sociología formal desconoce justamente este carácter histórico de la realidad histórica" ${ }^{\circ 7}$. 


\section{NOTAS}

${ }^{1}$ FICHTE, J.G., Los caracteres de la Edad Contemporánea, Revista de Occidente, Madrid, 1976, p. 79.

${ }^{2}$ FREYER, H., Introducción a la Sociología, Nueva Época, Madrid, 1951, p.99.

${ }^{3}$ Remitimos a algunos textos fundamentales: ZEITLIN, F., Ideología y teoría sociológica, Amorrortu, Buenos Aires, 1968; MARTINDALE, D., La teoría sociológica clásica, Aguilar, Madrid, 1979; GIDDENS, A., El capitalismo y la moderna teoría social, Labor, Barcelona, 1977; RITZER, G., Teoría sociológica clásica, McGraw Hill, Madrid, 1993; ARON, R., Las etapas del pensamiento sociológico, II, Siglo Veinte, Buenos Aires, 1970; SMELSER, N. J. - WARNER, R. S., Teoría sociológica. Análisis histórico y formal, Espasa Calpe, Madrid, 1984; MOYA, C., Sociólogos y sociología, Siglo XXI, Madrid, 1971; FERRAROTTI, F., El pensamiento sociológico de Auguste Comte a Max Horkheimer, Península, Barcelona, 1975; RICOEUR, P., Ideología y utopía, Gedisa, Barcelona, 1989; UÑA JUÁREZ, O., La sociología. Textos fundamentales, Libertarias, Madrid, 1.996; GINER, S., Historia del pensamiento social, Ariel, Barcelona, 1987

${ }^{4}$ Un análisis detenido y penetrante en este aspecto durkheimiano de sociología del conocimiento: TIRYAKIAN, E. A., Sociologismo y existencialismo. Dos enfoques sobre el individuo y la sociedad, Amorrortu, Buenos Aires, 1969, pp. 41-74 ("La sociedad según Durkheim") y bibliografía de y sobre Durkheim, pp. 241-242. Igualmente: TIRYAKIAN, E.A., "Emile Durkheim", en: BOTTOMORE, T. - NISBET, R., Historia del análisis sociológico, Amorrortu, Buenos Aires, 1988, pp. 218-272. Para las aportaciones de Durkheim a la sociología del conocimiento: DUVIGNAUD, J., Sociología del conocimiento, FCE, México, 1982; GONZÁLEZ GARCÍA, J.M., La sociología del conocimiento, hoy, Espejo, Madrid. 1979; STARK, W., La sociología del conocimiento, Morata, Madrid, 1963; GURVITCH, G. - MERTON. R.K., Sociología del conocimiento, Deucalión, Buenos Aires, 1953.

${ }^{5}$ Pensamiento que se concreta especialmente en: Las formas elementales de la vida religiosa, Akal, Madrid, 1982, p. 1 y ss. ("Introducción" y "Cuestiones preliminares"), sugerencias y estudio de R. Ramos. Sobre ello: BERGER, P.L. LUCKMANN, Th., La construcción social de la realidad, Amorrortu, Buenos Aires, 1972, p. 97 y ss., 133 y ss.

${ }^{6}$ Cf. DURKHEIM, E., La división del trabajo social, Akal, Madrid, 1982, p. 333 y ss. Muy acertadas reflexiones sobre la sociología en Durkheim de L. Rodríguez Zúñiga en el "estudio preliminar", p. XXXVII y ss. del citado volumen.

${ }^{7}$ Para una ampliación de la concepción durkheimiana de la sociedad y de la sociología misma: ZEITLIN, I., Ideología..., 265-316; MARTINDALE, D., La teoría..., pp. 101-104; ARON. R., Las etapas..., o.c., pp. 21-116; GIDDENS, A., El 
capitalismo..., pp. 127-202; RITZER, G., Teoría sociológica clásica..., pp. 205243; SMELSER, N.J. - WARNER, R.S., Teoría..., pp. 97-122; ABRAHAMSON, M., Sociological Theory, Prentice Hall, Englewood Cliffs, 1981, p. 66 y ss., 189 y ss., 232 y ss. ("The nature and form of sociological theory"); LEMERT, Ch. (ed.), Social Theory, Westview Press, Boulder-Oxford, 1993, pp. 77-108; ABRAHAM, M.F., Modern Sociological Theory, Oxford University Press, 1982, p. 175 y ss.; LUKES, S., Emile Durkheim: su vida y su obra, CIS, Madrid, 1984 ${ }^{8}$ DURKHEIM, E., Las reglas del método sociológico, Morata, Madrid, 1978, p. 35. ${ }^{9}$ ID., Ibid., p. 34. Otras obras de Durkheim: Education et sociologie, PUF, Paris, 1966; El socialismo, Akal, Madrid, 1987; El suicidio, Akal, Madrid, 1982; L'education morale, Alcan. Paris, 1923; Lecciones de sociología, Schapire, Buenos Aires, 1974.

${ }^{10}$ MOYA, C., Sociólogos..., p. 91.

${ }^{11}$ ID., Ibid., p. 97.

${ }^{12}$ ID., Ibid., p. 64.

${ }^{13}$ ID., Ibid., p. 101. Sobre Durkheim: DUVIGNAUD, J., Durkheim, sa vie et son cuvre, PUF, Paris, 1965; FENTON, S. (ed.), Durkheim and Modern Sociology, Cambridge University Press, London, 1984; GANE, M. (ed.), Radical Sociology of Durkheim and Mauss, Routledge and Kegan, London, 1992; GEHLKE, Ch. E., Emile Durkheim's Contributions to sociological Theory, Columbia University Press, New York, 1915; GIDDENS, A., Durkheim, Fontana Collins, Glasgow, 1981; FILLOUX, J.C., Durkheim et le socialisme, Genève, Droz, 1977; HIRST, Q.P., Durkheim. Bernard and epistemology, Routledge and Kegan, London, 1975; BOSSERMANN, P., "Georges Gurvitch et les durkheimiens, en France, avant et aprés la seconde guerre mondiale", Cahiers Intemationaux de Sociologie, 70 (1981), pp. 11-26; GURVITCH, G., La vocation actuelle de la sociologie, PUF, Paris, 1950; LACROIX, B., Durkheim et le politique, Presses Université de Montreal, Montreal, 1987; LEHMANN, J.M., Deconstructing Durkheim. A Post-Structuralism Critique, Routledge and Kegan, London, 1993; NISBET, R., Emile Durkheim, Prentice Hall, Englewood Cliffs, 1965; ID., The sociology of Emile Durkheim, Heinemann, London, 1975; PEARCE, F., The Radical Durkheim, Routledge and Kegan, London, 1989; TURNER, S. (ed.), Emile Durkheim. Sociologist and Moralist, Routledge and Kegan, London, 1993; WOLFF, K.H., Emile Durkheim: 1858-1917: A Collection of Essays With Translations and a Bibliography, Ohio State University Press, Glunlus, 1960; GURVITCH, G., "Pour le Centenaire de la Naissance de Durkheim", Cahiers Intemationaux de Sociologie, XXVII, 6 (1959), pp. 3 - 10; SELVIN, H.C., "Durkheim's Suicide and problems of empirical research", American Journal of Sociology, LXII, 6 (1958). pp. 607-619; UÑA JUÁREZ, O., "Sociología del suicidio. Ampliaciones epistemológicas", Psicopatología, 5,2 (1985), pp. 129-136.

${ }^{14}$ ARON, R., Las etapas..., p. 17. De interés las relaciones individuo-sociedadEstado: DURKHEIM, E., Lecciones de..., p. 44 y ss. 
${ }^{15}$ DILTHEY, W., Introducción a las ciencias del espiritu, Revista de Occidente, Madrid, 1966, p. 155. Sobre ello: ORTEGA Y GASSET, J., Kant. Hegel. Dilthey, Revista de Occidente, Madrid, 1961.

${ }^{16}$ DILTHEY, W., o.c., p. 548, también, p. 85.

${ }^{17}$ RICKERT, H., Introducción a los problemas de la filosofía de la historia, Nova, Buenos Aires, 1961, espec. pp. 23-90 ("La lógica de la ciencia histórica", donde se desarrollan los conceptos de "naturaleza", "espíritu", "método individualizador" y "valores en la historia"). Así como la clásica obra: Ciencia cultural y ciencia natural, Espasa Calpe, Madrid. 1965. Sobre el pensamiento del neokantismo: KLIMKE, F. - COLOMER, E., Historia de la filosofía, Labor, Barcelona, 1961, pp. 589-599 ("La vuelta a Kant en el Neokantismo").

${ }^{18}$ MOYA, C., Teoría..., p. 63. De interés al respecto: HUGUES, H.S., Conciencia y sociedad: la reorientación del pensamiento social europeo, 1890-1930, Aguilar, Madrid, 1972.

${ }^{19}$ Exposiciones sucintas del pensamiento de Weber: SMELSER, N.J. - WARNER, R.S., Teoría..., p. 123 y ss.; GIDDENS, A., El capitalismo..., p. 205 y ss.; RITZER, G., Teoría sociológica clásica, p. 45 y ss.; ARON, R., Las etapas..., p. 233 y ss.; MARTINDALE, D., La teoría..., P. 440 y ss.; ZEITLIN, L., Ideología..., p. 127 y ss.; MOYA, C., Sociólogos..., p. 113 y ss.; GINER, S., Historia..., p. 554 y ss.; ABRAHAMSON, M., Sociological..., p. 106 y ss., 126 y ss.; LEMERT, Ch. (ed.), Social theory..., p. 109 y ss.

${ }^{20}$ WEBER, M., Sobre la teoría de las ciencias sociales, Península, Barcelona, 1971, p. 6. Como elencos bibliográficos de y sobre Weber: GIDDENS, A., Política y sociología en Max Weber, Alianza, Madrid, 1972, pp. 93-98; FREUND, J., Sociología de Max Weber, Península, Barcelona, 1986, pp. 257-259; ARON, R., Las etapas..., II, pp. 314-316; LLANO, R., La sociología comprensiva como teoría de la cultura, CSIC, Madrid, 1992, pp. 540-567; UÑA JUÁREZ, O., "Introducción a la epistemología y metodología en Max Weber", Estudio Agustiniano, XIV (1979), pp. 505-519. Señalamos igualmente algunas versiones españolas de la obra de Weber: Economía y sociedad, FCE, México, 1985; La ética protestante y el espíritu del capitalismo, Península, Barcelona. 1989; El problema de la irracionalidad en las Ciencias Sociales, Tecnos, Madrid, 1985; Sobre la teoría de las Ciencias Sociales, Península, Barcelona, 1971; El político y el científico, Alianza, Madrid, 1988; Ensayos sobre metodología sociológica, Amorrortu, Buenos Aires, 1978; La acción social: ensayos metodológicos, Península, Barcelona, 1984; La ciudad, La Piqueta, Madrid, 1987; Ensayos de sociología contemporánea, Martínez Roca, Barcelona, 1972; Ensayos sobre Sociología de la religión, I-III, Taurus, Madrid, 1987-88; Política y ciencia, La Pléyade, Buenos Aires, 1976; ¿Qué es la democracia?, Leviatán, Buenos Aires, 1985; Escritos políticos, I-II, Folios, México, 1982; Estructuras de poder, Leviatán, Buenos Aires, 1985; Historia económica general, FCE, México, 1983; Ensayos 
de sociología contemporánea, ed. por GERTH, H.Y. y MILLS, C.W., Martínez Roca, Barcelona, 1972.

${ }^{21}$ Además de los escritos señalados sobre metodología, notamos el volumen que recoge varios escritos weberianos de este temario: WEBER, M., Il metodo delle scienze storico-sociali, Einaudi, Torino, 1992. Incluye los escritos sobre la "lógica de las ciencias de la cultura", pp. 143-237.

${ }^{22}$ WEBER. M., Sobre la teoría..., p. 42.

${ }^{23}$ ID., Ibid., p. 48.

${ }^{24}$ WEBER, M., La acción social: ensayos metodológicos..., p. 23.

${ }^{25}$ WEBER, M.. Economía y ..., p. 9.

${ }^{26}$ Cf. FREUND, J., Sociología..., p. 84.

${ }^{27}$ Sobre los tipos de verificación: WEBER, M., Economía..., p. 10.

${ }^{28}$ WEBER, M., Sobre la teoría..., p. 59.

${ }^{29}$ ID., Ibid., p. 74.

${ }^{30}$ WEBER, M., La acción social..., p. 172.

${ }^{31}$ BELTRAN, M., "Acerca de Weber y su ciencia exenta de valoraciones", en: JIMÉNEZ BLANCO, J. - MOYA VALGAÑON, C. (eds.), Teoría sociológica..., p. 409.

${ }^{32}$ WEBER, M., Sobre la teoría..., p. 9.

${ }^{33}$ ID., Ibid., p. 72.

${ }^{34}$ ID., Ibid., p. 9.

${ }^{35}$ ID., Ibid., p. 15.

${ }^{36}$ ID., Ibid., p. 19.

${ }^{37}$ ID., Ibid., p. 120.

${ }^{38}$ ID., Ibid., pp. 156-157.

${ }^{39}$ Cf. FREUND, J., Sociología..., p. 79.

${ }^{40}$ WEBER. M., Economía..., pp. 68-81. Señalamos algunas obras más significativas y específicas sobre Max Weber y los problemas aquí acometidos: BENDIX, R., Max Weber. An intellectual portrait, Doubleday, New York, 1960; BENDIX, R. - ROTH, G., Scholarship and partisanship: Essays on Max Weber, University of California Press, Berkeley, 1971; BRENNAN, C.M., Max Weber on Power and Social Stratification. An Interpretation and Critique, Amesbury, London, 1993; BRUBAKER, R., The Limits of Rationality, Routledge and Kegan, London, 1984; ELDRIDGE, J.E.T., Max Weber. The Interpretation to Social Reality, Michael Joseph. London, 1971; GERTH, H.H. - MILLS, C.W., From Max Weber. Essays on Sociology, Oxford University Press, Oxford, 1958; ID., El capitalismo y la moderna teoría social, Labor, Barcelona, 1985, pp. 205-299; HOLTON, R. - TURNER, B.S., Max Weber on Economy and Society, Routledge and Kegan, London, 1990; IZQUIERDO, J. de D., Max Weber. Precedentes y claves metodológicas, Publicaciones de la Universidad de Castilla-La Mancha, Cuenca, 1991; JANOSKA-BENDL. J., Max Weber y la sociología de la Historia, 
Sur, Buenos Aires, 1972; JIMÉNEZ BLANCO, J., "Weber, Schütz y Garfinkel sobre racionalidad", en: JIMÉNEZ BLANCO, J. - MOYA VALGAÑÓN, C. (eds.), Teoría sociológica..., pp. 366-393; LÖWITH, K., Max Weber and Karl Marx, Routledge and Kegan, London, 1993; MARSAL, J.F., Conocer Max Weber y su obra, Dopesa, Barcelona, 1978; MITZMAN, A., La jaula de hierro: Una interpretación histórica de Max Weber, Alianza, Madrid. 1969; RUNCIMAN, W.G., A Critique of Max Weber's Philosophy of Social Science, Cambridge University Press, London, 1972; SAHAY, A. (ed.), Max Weber and Modern Sociology, Routledge and Kegan, London, 1971; SAYER, D., Capitalism and Modernity, MacMillan, London, 1990; SAZBON, J. (ed.), Presencia de Max Weber, Nueva Visión, Buenos Aires, 1971; STAMMER, O. (ed.), Max Weber and Sociology Today, Harper \& Row, New York, 1972; TURNER, B.S., Max Weber: From History to Modernity, Routledge and Kegan, London, 1992; TURNER, Ch., Modernity and Politics in the Work of Max Weber, Routledge and Kegan, London, 1992; VINCENT. J.M., La metodología de Max Weber, Anagrama, Barcelona, 1972; WRONG. D., (ed.), Max Weber, Prentice-Hall, Englewood Cliffs, 1970; MOYA, C., "Repensando a Weber", en: MOYA. C. y otros (eds.), Escritos de teoría sociológica..., pp. 765-775.

${ }^{41}$ Las etapas..., II, p. 12. Bibliografía de y sobre Pareto: PARETO, V., Forma y equilibrio sociales, Alianza, Madrid, 1980, pp. 44-51; ARON, R., Las etapas..., pp. 229-231; FERRAROTTI, F., El pensamiento sociológico..., p. 211-215.

${ }^{42}$ ARON, R., Las etapas..., p. 15.

${ }^{43}$ ID., Ibid,. p. 13. Exposiciones de su pensamiento, en: GINSBERG, M., Ensayos de sociología y filosofía social, Aguilar, Madrid, 1961, pp. 352 - 379; FERRAROTTI, F., El pensamiento sociológico..., pp. 185-215; ARON. R.. Las etapas..., o.c., pp. 125-231; BELLAMY, R.. Modern Italian Social Theory, Polity Press, Oxford, 1987, pp. 12-33; DUNCAN MITCHELL, G., Historia..., I, pp. 178-192; HUGUES, H.S., Conciencia y sociedad..., pp. 58-61 y 183-198; RODRÍGUEZ IBAÑEZ, J.E., La perspectiva..., p. 144 y ss.; PINO ARTACHO, J. del, La teoría..., p. 102 y ss.; SCHUMPETER, J.A., Diez grandes economistas: de Marx a Keynes, Alianza, Madrid, 1971, pp. 159-197.

${ }^{44}$ Cf. PARETO, V., Escritos sociológicos, Alianza, Madrid, 1987, p. 287 (parágrafo 150 del Tratado de sociología).

${ }^{45}$ ID., Ibid., p. 288 (párrafo 151 del Tratado...).

${ }^{46}$ PARETO, V., Forma..., p. 151 (parágrafo 2229 del Tratado...).

${ }^{47}$ Remitimos a la bibliografía de Pareto: Les Systèmes Socialistes, I-II, Giard y Briére, Paris, 1902-1903; Fatti et Teorie, Vallecchi, Firenze, 1920; The Mind and Society. traducción del Trattato di sociologia generale, I-IV, Harcourt, Brace and Co., New York, 1935. Sobre Pareto: BORKENAU, F., Pareto, FCE, México, 1978; BOUSQUET, G.H., The Work of Pareto, The Sociological Press, Hannover, N.H., 1928; ID., Vilfredo Pareto: sa vie et son ceuvre, Payot, Paris, 1928; 
HANDMAN, M.S., "The Sociological Method of Vilfredo Pareto", en: RICE, S.A. (ed.), Methods in Social Science, University of Chicago Press, Chicago, 1931, pp. 139-153; HENDERSON, L.J., Pareto General Sociology. A Physiologist's Interpretation, Harvard University Press, Cambridge, 1935; HOMANS, G.C. - CURTIS, C.P., An Introduction to Pareto; His Sociology, Knopf, New York, 1934; FINER, S.E. (ed.), Vilfredo Pareto: Sociological Writings, F.A. Praeger, New York, 1966; MEISEL, J.H., Pareto and Mosca, Prentice Hall, New Jersey, 1965; PERRIN, G., Sociologie de Pareto, PUF, Paris, 1966. Para una crítica del irracionalismo paretiano, entre otros: LUKACS, G., El asalto a la razón, Grijalbo, Barcelona, 1968; GONZÁLEZ GARCÍA, J.M., La sociología del conocimiento, hoy, Espejo, Madrid, 1979.

48 "Prólogo: Ferdinand Tönnies y la ciencia social moderna", de S. Giner y L Flaquer, en: TÖNNIES, F.. Comunidad y asociación, Península, Barcelona, 1979, pp. 522. De Tönnies, aquí, especialmente, el libro primero: "Presentación general de los conceptos fundamentales", p. 25 y ss. Ampliamente sobre la sociología como ciencia y como disciplina: On Sociology pure and applied, ed. by W.J. Cahnman and R. Heberlee, Chicago University Press, 1971. De Tönnies igualmente: Costom. An Essay on Social Codes, Free Press, Glencoe, 1961. Las expresiones calificativas que él emplea en alemán para la división de la sociología son: "reine" y "theoretische", "angewandte" y "empirische".

${ }^{49}$ FREYER, H., Introducción a la sociología, Nueva Época, Madrid, 1951, p 110. Sobre Tönnies: GINSBERG, M., Ensayos de sociología..., p. 382 y ss; CAHNMAN, W.J. (ed.), Ferdinand Tönnies. A New Evaluation. Essay and Documents, E.J. Brill, Leiden, 1973 (que incluye una colaboración de Parsons sobre el concepto de "comunidad"); WIRTH, L., "The Sociology of Ferdinand Tönnies", American Journal of Sociology, XX (1926), pp. 412-422; GINER, S., El progreso de la conciencia sociológica, Península. Barcelona, 1974, pp. 6196; FREUND, J., "La sociología alemana en la época de Max Weber", en: BOTTOMORE, T. - NISBET, R.. Historia del análisis..., pp. 178 y ss.; MARTINDALE, D., La teoría..., p. 95 y ss.; DUNCAN MITCHELL, G.. Historia..., p. 162 y ss.

${ }^{50}$ SOMBART, W., "Sociología. Un prólogo", en: Noosociología, Instituto de Estudios Políticos, Madrid, 1962, p. 3.

${ }^{51}$ SOMBART, W., Ibid., p. 2.

52 SOMBART, W., "La comprensión", en: Noosociología, p. 117. Sobre el pensamiento de Sombart, sus obras, y estudios varios sobre él, remitimos a: GODELIER, M., Racionalidad e irracionalidad en la economía, Siglo XXI, Madrid. 1974.

${ }^{53}$ ID., Ibid., p. 119.

${ }^{54}$ ID., Ibid., p. 120.

${ }^{55}$ SIMMEL, G., Sociología. Estudios sobre las formas de socialización, I, 
Revista de Occidente, Madrid, 1977, p. 23. “...no contiene la Sociología ningún objeto que no esté tratado ya en las ciencias existentes, sino que es sólo un nuevo camino para todas ellas, un método científico que, justamente por ser aplicable a la totalidad de los problemas, no constituye una ciencia por sí" (Ibid., p. 14). “... la Sociología podría constituir una ciencia particular, hallando su objeto una nueva línea trazada a través de hechos que, como tales, son perfectamente conocidos; sólo que, sin haberles sido hasta entonces aplicado el concepto que descubre el aspecto de estos hechos correspondiente a aquella línea, haciéndole constituir una unidad metódica y científica común a todos" (Ibid., p. 15).

${ }^{56}$ ID., Ibid., p. 15. Exposiciones del pensamiento de Simmel: MARTINDALE, D., La teoría..., p. 275 y ss.; GINSBERG, M., Ensayos de sociología..., p. 382 y ss.; FREUND, J., "La sociología alemana...", p. 186 y ss.; DUNCAN MITCHELL, G., Historia..., I, p. 166 y ss.; RITZER, G., Teoría sociológica clásica, pp. 299333; ABRAHAMSON, M.. Sociological..., pp. 154-168. Para las relaciones de Simmel con otras corrientes de pensamiento en la sociología: LEGER, F., "Georg Simmel and Max Weber", Social Science Information, 25 (1986), pp. 881-898; LEVINE, D., Simmel and Parsons, Arno Press, New York, 1957; WEINSTEIN, D. - WEINSTEIN, M., Postmodern (ized) Simmel, Routledge and Kegan, London, 1993

${ }^{57}$ SIMMEL, G., Sociología..., I, p. 15. Otros escritos del pensador de Berlín: Conflict and the Web of Group Affiliations, The Free Press, Glencoe, 1955: Cultura femenina y otros ensayos, Espasa Calpe, Madrid, 1938; El individuo y la libertad, Península, Barcelona, 1986; On Women. Sexuality and Love, Yale University Press, New Haven, 1984; Georg Simmel: Sociologist and European, ed. by P. Lawrence, Nelson, Sunbury on Thames, 1976; La filosofía del dinero, Revista de Occidente, Madrid, 1977; ID., On Individuality and Social Forms, ed. by D.N. Levine, The University of Chicago, Chicago \& London, 1971; "The sociology of Possibility", American Journal of Sociology, LV (1949). pp. 254-261.

${ }^{58}$ Sobre Simmel: COSER, L., Georg Simmel, Prentice Hall, New Jersey, 1965; FRISBY, D., Georg Simmel, Ellis Harwood, London \& New York, 1984; ID., Sociological Impressionism: A Reassessment of Georg Simmel's Social Theory, Heinemann, London, 1981; ID., Simmel and science, Routledge and Kegan, London, 1992; GERHARDS, J., "Georg Simmel's Contribution of Theory of Emotions", Social Science Information, 5 (1986), pp. 901-922; WEINGARTNER, R.H., Experience and Culture: The Philosophy of Georg Simmel, Wesleyan University Press, Middletown, 1962; WOLFF. K.H. (ed.), The Sociology of Georg Simmel, The Free Press, Glencoe, 1950.

${ }^{59}$ Cf. SCHELER, M.,Sociología del saber, Siglo Veinte, Buenos Aires, 1973. p. 10. ${ }^{60}$ ID., Ibid., p. 10. Como exposiciones del pensamiento de M. Scheler: HIRSCHBERGER, J., Historia de la filosofia, II, Herder, Barcelona, 1965, pp. 359-364; KLIMKE, F. - COLOMER. E., Historia de la filosofía, Labor, Barcelona, 1961, pp. 773-785; MARÍAS, J., Historia de la filosofía, Revista de Occidente, 
Madrid, 1971, pp. 410-411; DUPUY, M., La philosophie de Max Scheler. Son évolution et son unité, I-II, PUF, Paris, 1959; STAUDE. J.R., Max Scheler. An intellectual portrait, The Free Press, New York, 1967; PINTOR RAMOS, A., El humanismo de Max Scheler, Ed. Católica, Madrid, 1978.

${ }^{61}$ SCHELER, M., Sociología..., p. 13. Las aportaciones del autor sobre sociología se reúnen en: Schriften zur Soziologie und Weltanschauungslehre, Francke, Bern-München, 1963, 455 pp. (Gesammelte Werke, VI).

${ }^{62}$ GONZÁLEZ GARCÍA, J.M., La sociología del conocimiento, hoy, Espejo, Madrid, 1979, pp. 23-61; HUGUES, H.S., Conciencia y sociedad, Aguilar, Madrid, 1972, pp. 25-49; FILIPPONE-THAULERO, V., Società e cultura nel pensiero di Max Scheler, I-II, Giufré, Milano, 1964-1969. Una amplia y sistemática bibliografía, en: PINTOR RAMOS, A., El humanismo..., pp. XV - XXVIII.

${ }^{63}$ SCHELER, M., Sociología..., pp. 63-64.

${ }^{64}$ Cf. Ideología y utopía, Aguilar, Madrid, 1966, p. 395.

65 "El objeto de la sociología teorética, en su opinión, es la ordenación y sistematización del tejido entero de las relaciones entre seres humanos" Cf. GINSBERG. M., Ensayos de sociología..., p. 386. Sobre el pensamiento de von Wiese: MARTINDALE, D., La teoría..., p. 299 y ss.; DUNCAN MITCHELL, G., Historia..., I, p. 173 y ss.; FREYER. H., Introducción..., p. 111 y ss.; KÖNIG, R., "Germany", en: ROUCEK, J.S. (ed. and dir.), Contemporary Sociology, Philosophy Library, New York, 1958, pp. 779-806; ABEL. Th., Systematic Sociology in Germany, Columbia University Press, New York, 1929; SIAUFFER, E., La méthode relationnelle en psychologie sociale et en sociologie selon L. von Wiese, Delachaux et Niestlé, Paris, 1950.

${ }^{66}$ WIESE, L. von, System der allgemeinen Soziologie als Lehre von den sozialen Prozessen und den sozialen Gebilden der Menschen (Beziehungslehre), Duncker und Humblot, München-Leipzig, 1933, p. 101. Como textos más accesibles: Sociología. Su historia y sus problemas, Labor, Barcelona, 1932; Systematic Sociology, ed. by H. Becker, John Wiley, New York, 1932.

${ }^{67}$ Cf. Introducción..., p. 112. Para la sociología de la "época" weberiana siempre es actual el excelente escrito: ARON, R., La sociología alemana contemporánea, Paidós, Buenos Aires, 1965. 